\title{
Reverse Engineering for Reduction Parallelization via Semiring Polynomials
}

\author{
Akimasa Morihata \\ Graduate School of Arts and Sciences, \\ the University of Tokyo \\ Tokyo, Japan \\ morihata@graco.c.u-tokyo.ac.jp
}

\author{
Shigeyuki Sato \\ Graduate School of Information Science and Technology, \\ the University of Tokyo \\ Tokyo, Japan \\ sato.shigeyuki@mi.u-tokyo.ac.jp
}

\begin{abstract}
Parallel reduction, which summarizes a given dataset, e.g., the total, average, and maximum, plays a crucial role in parallel programming. This paper presents a new approach, reverse engineering, to automatically discovering nontrivial parallel reductions in sequential programs. The body of the sequential reduction loop is regarded as a black box, and its input-output behaviors are sampled. If the behaviors correspond to a set of linear polynomials over a semiring, a divide-and-conquer parallel reduction is generated. Auxiliary reverse-engineering methods enable a long and nested loop body to be decomposed, which makes our parallelization scheme applicable to various types of reduction loops. This approach is not only simple and efficient but also agnostic to the details of the input program. Its potential is demonstrated through several use case scenarios. A proof-of-concept implementation successfully inferred linear polynomials for nearly all of the 74 benchmarks exhaustively collected from the literature. These characteristics and experimental results demonstrate the promise of the proposed approach, despite its inherent unsoundness.
\end{abstract}

CCS Concepts: • Computing methodologies $\rightarrow$ Parallel programming languages; • Software and its engineering $\rightarrow$ Automatic programming; Software testing and debugging.

Keywords: parallelization, reduction loop, reverse engineering, semiring, program synthesis

\section{ACM Reference Format:}

Akimasa Morihata and Shigeyuki Sato. 2021. Reverse Engineering for Reduction Parallelization via Semiring Polynomials. In Proceedings of the 42nd ACM SIGPLAN International Conference on Programming Language Design and Implementation (PLDI '21), June

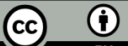

This work is licensed under a Creative Commons Attribution 4.0 International License.

PLDI '21, fune 20-25, 2021, Virtual, Canada

() 2021 Copyright held by the owner/author(s).

ACM ISBN 978-1-4503-8391-2/21/06.

https://doi.org/10.1145/3453483.3454079

20-25, 2021, Virtual, Canada. ACM, New York, NY, USA, 15 pages. https://doi.org/10.1145/3453483.3454079

\section{Introduction}

Parallel reduction (also known as parallel aggregation) summarizes a given dataset, e.g., the total, average, and maximum. It plays a crucial role in parallel programming and is used particularly for preparing inputs for and aggregating results from independent tasks. Most parallel programming frameworks and languages support parallel reduction.

Despite the support for parallel reduction, parallelizing nontrivial reductions is still difficult. Consider, for example, the reduction loops defined in Figures 1 and $2^{1}$. They do not match the typical parallel reduction patterns. Parallelizing them requires the development of equivalent programs, each consisting of only an associative operator. This difficulty has led to numerous studies on automatically discovering nontrivial parallel reductions from sequential programs [7$11,15,18,22,25,27,28,31,36,38]$.

There are four desirable properties for a reduction parallelization method: it should be simple, efficient, powerful enough to parallelize a wide range of reductions, and agnostic to the details and variations of the given sequential program. Unfortunately, no existing method acquires all the properties. Those based on symbolic analysis $[15,18,22,25,31,36,38]$ are commonly sensitive to the use of reduction operators. For instance, they tend to have difficulty parallelizing loops containing conditionals for calculating maximums like the one defined in Figure 2. Those based on solver-aided program synthesis [7-9, 11, 27, 28, 31] are

\footnotetext{
${ }^{1}$ All programs here are written in Python.

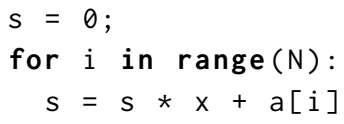

Figure 1. Reduction loop

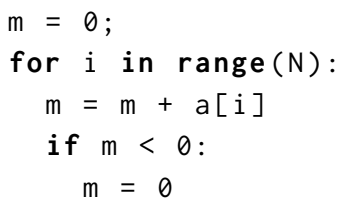
for calculating a power series on the basis of Horner's method.
Figure 2. Reduction loop for calculating maximum suffix sum. 


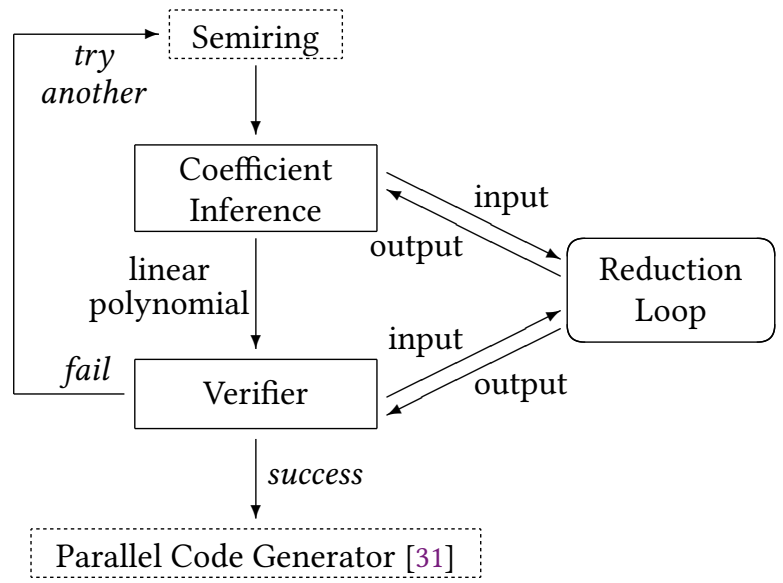

Figure 3. Overview of the Proposed Approach

relatively tolerant of code variation but rather complicated and costly. Besides, the use of a constraint solver generally induces fragility against practical dirty code, which contain fragments hard to translate to constraint formulae, such as external function calls and indirect memory accesses.

We present a new approach to automatic reduction parallelization: reverse engineering. In reverse engineering, we analyze a black-box system, whose internal structure is not accessible, by carefully probing its behaviors. Following this paradigm, we intentionally regard the given sequential program as a black box and compare its input-output behaviors with parallelizable patterns. This approach is an extreme trade-off. It sacrifices potentially valuable information inside the program code and is unsound because of the gap between the program semantics and finite observations. Instead, it has two ideal characteristics in nature: insensibility to code variations and simplicity owing to its concentration on input-output behaviors.

We demonstrate that our reverse-engineering approach to reduction parallelization, depicted in Figure 3, can be efficient and powerful. The approach is guided by the following two key findings. First, reverse engineering brings the full potential of semiring-based reduction parallelization [15, 22, 25, 31, 38], which models the loop body in terms of linear polynomials ${ }^{2}$ over semirings and leverages the associativity inside the polynomials for divide-and-conquer parallel reduction. Reverse engineering reduces the weakness of existing semiring-based methods, which requires programs to be written using semiring operators. Second, reverse engineering is efficient. Candidate linear polynomials, particularly their coefficients, can be immediately inferred by feeding carefully-prepared inputs to the loop body. Random testing can quickly reject unsuitable candidates.

\footnotetext{
${ }^{2}$ The term "linear" is used here only for the linearity of polynomials, not for the single-useness of variables.
}

Consider the loop defined in Figure 1 by assuming that the loop body, i.e., the computation of updating reduction variable $\mathrm{s}$, is equivalent to polynomial $a_{0}+a_{1} \times \mathrm{s}$, in which $a_{0}$ and $a_{1}$ are coefficients and $\mathrm{s}$ is an indeterminate. We observe the following input-output behaviors of the loop body, in which the LHS and RHS respectively denote the states before and after the loop body execution. The RHS includes only the updated value, i.e., the value of $s$.

$$
\begin{aligned}
& \{s=0, x=10, a[i]=3\} \rightarrow\{s=3\} \\
& \{s=1, x=10, a[i]=3\} \rightarrow\{s=13\} \\
& \{s=2, x=10, a[i]=3\} \rightarrow\{s=23\} \\
& \{s=0, x=15, a[i]=7\} \rightarrow\{s=7\} \\
& \{s=1, x=15, a[i]=7\} \rightarrow\{s=22\}
\end{aligned}
$$

It is natural to anticipate that $a_{1}=\mathrm{x}$ and $a_{0}=\mathrm{a}$ [i]. Although this anticipation may not be correct, hundreds of rounds of random testing may convince us that there is a high probability that it is correct.

The flexibility of choosing an appropriate semiring is crucial for parallelizing various types of reduction loops. This is evidenced by the following input-output behaviors of the loop body defined in Figure 2.

$$
\begin{array}{ll}
\{m=-\infty, a[i]=0\} & \rightarrow\{m=0\} \\
\{m=-\infty, a[i]=10\} & \rightarrow\{m=0\} \\
\{m=0, a[i]=10\} & \rightarrow\{m=10\} \\
\{m=5, a[i]=10\} & \rightarrow\{m=15\} \\
\{m=5, a[i]=15\} & \rightarrow\{m=20\}
\end{array}
$$

While these behaviors cannot be expressed by linear polynomial $a_{0}+a_{1} \times \mathrm{m}$, they can be accounted for a linear polynomial, $\max (0, a[i]+m)$, over another semiring consisting of the binary maximum and the addition.

We also present auxiliary reverse-engineering methods we developed for decomposing a long loop body and a loop nest. They enable our parallelization approach to be applied to minimal fragments of loop-free code, and the discovered parallel reductions are combined into a large parallel reduction. They thus make our parallelization scheme scalable in terms of input program size.

We demonstrated the powerfulness and efficiency of our approach through experiments based on 74 benchmark problems exhaustively collected from the literature [7-10, 18, 28, 31, 36] on automatic parallelization of complex reductions. Our proof-of-concept implementation successfully inferred linear polynomials for all the benchmarks except for a few with unimplemented semirings.

Our work makes the first step of the reverse-engineering approach to reduction parallelization. Further development is necessary for incorporating the proposed approach into full-fledged parallelizing compilers mainly because of the inherent unsoundness. The possibility of a pathological case that contradicts observed input-output behaviors generally 
cannot be disproved only with the proposed approach. Nevertheless, we believe that our approach is useful and promising in many use case scenarios.

Our main contributions are summarized as follows:

- We have established a new reverse-engineering approach to reduction parallelization (Section 3).

- We have developed auxiliary reverse-engineering methods for decomposing a long loop body and a loop nest to make our parallelization scheme applicable to various types of reduction loops (Section 4).

- We demonstrate the potential applicability of our reverse-engineering approach through different use case scenarios (Section 5).

- We experimentally demonstrated the powerfulness and efficiency of our parallelization scheme for 74 benchmarks collected from the literature (Section 6).

\section{Parallel Reduction by Simplifying Linear Polynomials over Semiring}

This section reviews the existing approach that uses linear polynomials over a semiring for reduction parallelization [22, $25,31,38]$.

\subsection{Semiring and Linear Polynomials}

A semiring generalizes a pair of closely related algebraic operators such as $(+, \times)$ and $(\cup, \cap)$. Formally, a semiring is a five-tuple, $(S, \oplus, \otimes, \overline{0}, \overline{1})$, where $S$ is a set of values, $\oplus$ and $\otimes$ are binary operators over $S$, and $\overline{0}$ and $\overline{1}$ are elements of $S$. They should satisfy the following properties.

$$
\begin{array}{ll}
a \oplus \overline{0}=\overline{0} \oplus a=a & \overline{0} \text { is the identity for } \oplus \\
a \oplus(b \oplus c)=(a \oplus b) \oplus c & \oplus \text { is associative } \\
a \oplus b=b \oplus a & \oplus \text { is commutative } \\
a \otimes \overline{1}=\overline{1} \otimes a=a & \overline{1} \text { is the identity for } \otimes \\
a \otimes(b \otimes c)=(a \otimes b) \otimes c & \otimes \text { is associative } \\
a \otimes(b \oplus c)=(a \otimes b) \oplus(a \otimes c) & \otimes \text { is left-distributive } \\
(b \oplus c) \otimes a=(b \otimes a) \oplus(c \otimes a) & \otimes \text { is right-distributive } \\
a \otimes \overline{0}=\overline{0} \otimes a=\overline{0} & \overline{0} \text { is the zero-element }
\end{array}
$$

Examples of semirings include addition and multiplication over integers $(\mathbb{Z},+, \times, 0,1)$, disjunction and conjunction over Boolean values $(\{0,1\}, \vee, \wedge, 0,1)$, binary minimum and binary maximum over integers with infinity $(\mathbb{Z} \cup\{\infty,-\infty\}$, $\min , \max , \infty,-\infty)$, binary maximum and additions over integers with negative infinity $(\mathbb{Z} \cup\{-\infty\}$, $\max ,+,-\infty, 0)$, binary maximum and multiplication over positive integers $\left(\mathbb{Z}_{+}, \max , \times, 0,1\right)$, and union and intersection over sets $\left(2^{S}, \cup, \cap, \emptyset, S\right)$. As seen in these examples, multiplication $\otimes$ is frequently commutative. Unless otherwise stated, we assume commutativity of $\otimes$. We write $(\oplus, \otimes)$ instead of $(S, \oplus, \otimes, \overline{0}, \overline{1})$ if the omitted components are obvious from the context or not important.

Given semiring $\mathcal{S}=(S, \oplus, \otimes, \overline{0}, \overline{1})$ and a set of indeterminates, $\left\{x_{1}, x_{2}, \ldots, x_{n}\right\}$, a linear polynomial over $\mathcal{S}$ is an expression of the following form, where $a_{0}, a_{1}, \ldots, a_{n} \in S$ are coefficients:

$$
a_{0} \oplus\left(a_{1} \otimes x_{1}\right) \oplus \cdots \oplus\left(a_{n} \otimes x_{n}\right) .
$$

\subsection{Parallelization}

A reduction loop is parallelizable if its body is equivalent to a set of linear polynomials. As an example, consider the following program for calculating the maximum sum of a consecutive subarray of array $[10,28]$.

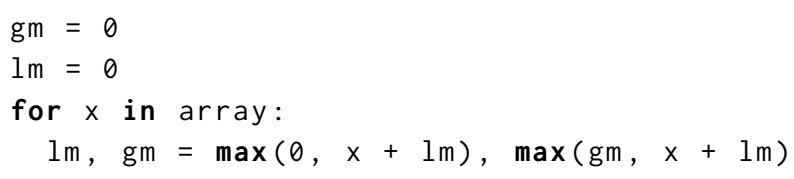

In this program, $1 \mathrm{~m}$ and gm are the reduction variables because of their loop-carried dependences ${ }^{3}$. They are simultaneously updated using Python's "target list assignment" syntax. We can express the loop body by the following pair of linear polynomials over $(\mathbb{Z} \cup\{-\infty\}, \uparrow,+,-\infty, 0)$, where the reduction variables correspond to the indeterminates, $l \mathrm{~m}$ and $\mathrm{gm}$, and $\uparrow$ denotes the binary infix maximum operator; hence, each polynomial is of the form of $a_{0} \uparrow\left(a_{1}+\operatorname{lm}\right) \uparrow\left(a_{2}+g m\right)$.

$$
\left(\begin{array}{c}
0 \uparrow(\mathrm{x}+\operatorname{lm}) \uparrow(-\infty+\mathrm{g} m), \\
-\infty \uparrow(\mathrm{x}+\operatorname{lm}) \uparrow(0+\mathrm{gm})
\end{array}\right)
$$

The former and latter polynomials respectively correspond to the computations for $1 \mathrm{~m}$ and gm.

The linear polynomials lead to a divide-and-conquer parallel reduction. Assume that a processor takes charge of consecutive iterations of the loop, say the $s$-th to $t$-th iterations (i.e., those corresponding to array $[s]$ to array $[t]$ ). Even without knowing the result of the $(s-1)$-th iteration, the processor can summarize the allocated loop iterations into linear polynomials (or, more precisely, their coefficients). For instance, let array $[s]$ and array $[s+1]$ be 3 and -4 , respectively; then, the summary of the $s$-th and $(s+1)$-th iterations is the following pair of linear polynomials.

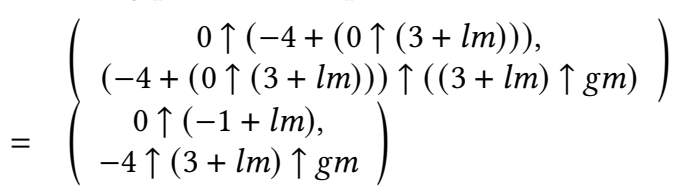

In this way, each processor calculates an iteration summary, i.e., a set of linear polynomials. The result for the reduction loop is then obtained by recursively merging the summaries and supplying the initial values to the reduction variables. The time complexity of this divide-and-conquer approach is $\mathrm{O}(N / p+\log p)$, where $N$ and $p$ are the numbers of loop iterations and processors, respectively.

\footnotetext{
${ }^{3}$ We do not regard loop counters (e.g., $x$ in this example) as reduction variables because their values can be determined without evaluating the loop.
} 


\section{Inferring Linear Polynomials over Semiring by Reverse Engineering}

The proposed approach combines reverse engineering with the one described in Section 2. The following loop is the target of parallelization.

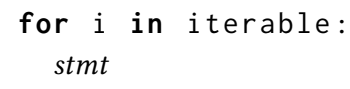

We assume that stmt contains no global side effects such as I/O and modification of global variables and that the execution of stmt does not modify iterable. Let $X$ be the set of variables that appear in stmt and $Y=\left\{y_{1}, y_{2} \ldots, y_{k}\right\} \subseteq X$ be a set of reduction variables. A standard dependence analysis can recognize the reduction variables. We will also propose a reverse-engineering approach to it in Section 4.1.

We use notation similar to Hoare logic to express the input-output behavior of stmt. Specifically,

$$
\left\{x_{i}=v_{i} \mid x_{i} \in X\right\} \text { stmt }\left\{x_{j}=v_{j}^{\prime}\right\}
$$

denotes the following input-output behavior: executing stmt under precondition $x_{0}=v_{0} \wedge x_{1}=v_{1} \wedge \cdots$ results in postcondition $x_{j}=v_{j}^{\prime}$. Note that this notation does not exclude the case in which a variable other than $x_{j}$ is updated.

\subsection{Overview}

Given a finite set of semirings, $\mathcal{S}_{\mathcal{R}}$, we use the following algorithm to find a subset of $\mathcal{S}_{\mathcal{R}}$ over which stmt corresponds to linear polynomials.

1. Let $R=\emptyset$.

2. For each $(\oplus, \otimes) \in \mathcal{S}_{\mathcal{R}}$ :

a. For each $y \in Y$, repeat the following test a sufficient number of times. If it fails, reject $(\oplus, \otimes)$ and go to Step 2.

i. Let $\mathcal{E}_{X}=\left\{x_{j}=v_{j} \quad \mid x_{j} \in X \backslash Y\right\}$ and $\mathcal{E}_{Y}=$ $\left\{y_{i}=w_{i} \mid y_{i} \in Y\right\}$, where each $v_{i}$ or $w_{i}$ is a randomly generated value, and find $w$ such that $\left(\mathcal{E}_{X} \cup \mathcal{E}_{Y}\right)$ stmt $\{y=w\}$ by executing stmt.

ii. By reverse engineering, generate a linear polynomial $a_{0} \oplus\left(a_{1} \otimes y_{1}\right) \oplus \cdots \oplus\left(a_{k} \otimes y_{k}\right)$ using $\mathcal{E}_{X}$.

iii. Check $a_{0} \oplus\left(a_{1} \otimes w_{1}\right) \oplus \cdots \oplus\left(a_{k} \otimes w_{k}\right)=w$.

b. Add $(\oplus, \otimes)$ to $R$

\section{Return $R$}

For each semiring in $\mathcal{S}_{\mathcal{R}}$ and each reduction variable, this algorithm tests whether a particular linear polynomial and the loop body have the same behavior. The process for generating a linear polynomial (Step 2(a)ii) depends on the semiring and will be described in Section 3.2. This algorithm tests all the semirings and identifies all the ones appropriate for the parallelization of this loop. It is useful for auxiliary methods presented in Sections 4.2 and 4.3.

\subsection{Method for Inferring Coefficients}

Now we present algorithms for inferring the coefficients of linear polynomial $a_{0} \oplus\left(a_{1} \otimes y_{1}\right) \oplus \cdots \oplus\left(a_{k} \otimes y_{k}\right)$ over semiring $(S, \oplus, \otimes, \overline{0}, \overline{1})$.

3.2.1 Inferring Constant Term. It is not difficult to determine $a_{0}$. Providing $\overline{0}$ to each $y_{i}$ should result in $a_{0}$ if the computation is equivalent to the linear polynomial. Formally, we infer $a_{0}$ by the following execution of stmt.

$$
\left(\mathcal{E}_{X} \cup\left\{y_{i}=\overline{0} \mid y_{i} \in Y\right\}\right) \text { stmt }\left\{y=a_{0}\right\}
$$

3.2.2 Semirings with Additive Inverses. The semiring is said to have additive inverses if for each $s \in S$ there exists $s^{-1} \in S$ such that $s \oplus s^{-1}=\overline{0}$. Many important semirings, including $(+, \times)$, have additive inverses.

Additive inverses enable us to determine each $a_{i}$. This is done by setting $y_{i}$ to $\overline{1}$ and the other reduction variables to $\overline{0}$, executing stmt, and extracting $a_{0}^{-1}$ from the output. Formally, $a_{i}=w \oplus a_{0}^{-1}$, where $w$ is obtained by executing:

$$
\left(\mathcal{E}_{X} \cup\left\{y_{i}=\overline{1}\right\} \cup\left\{y_{j}=\overline{0} \mid y_{j} \in Y \backslash\left\{y_{i}\right\}\right\}\right) \text { stmt }\{y=w\} \text {. }
$$

The following reasoning justifies this inference.

$$
\begin{aligned}
& \left(a_{0} \oplus\left(a_{1} \otimes \overline{0}\right) \oplus \cdots \oplus\left(a_{i} \otimes \overline{1}\right) \oplus \cdots \oplus\left(a_{k} \otimes \overline{0}\right)\right) \oplus a_{0}^{-1} \\
= & a_{0} \oplus a_{i} \oplus a_{0}^{-1} \\
= & a_{i} .
\end{aligned}
$$

3.2.3 Distributive Lattices. Sometimes the addition and multiplication in a semiring can exchange roles. This holds particularly common in semirings that are distributive lattices: $(\vee, \wedge)$ with $(\wedge, \vee),(\min , \max )$ with $(\max , \min )$, and $(\cup, \cap)$ with $(\cap, \cup)$.

Somewhat surprisingly, for these semirings, we can use $a_{0} \oplus a_{i}$ instead of $a_{i}$. Note that $\overline{1}$ is the zero-element for semiring $(S, \otimes, \oplus, \overline{1}, \overline{0})$, i.e., $a \oplus \overline{1}=\overline{1} \oplus a=\overline{1}$.

$$
\begin{aligned}
& a_{0} \oplus\left(\left(a_{0} \oplus a_{i}\right) \otimes y_{i}\right) \\
= & \left(a_{0} \otimes \overline{1}\right) \oplus\left(a_{0} \otimes y_{i}\right) \oplus\left(a_{i} \otimes y_{i}\right) \\
= & \left(a_{0} \otimes\left(\overline{1} \oplus y_{i}\right)\right) \oplus\left(a_{i} \otimes y_{i}\right) \\
= & \left(a_{0} \otimes \overline{1}\right) \oplus\left(a_{i} \otimes y_{i}\right) \\
= & a_{0} \oplus\left(a_{i} \otimes y_{i}\right)
\end{aligned}
$$

Therefore, we can obtain $a_{i}$ by executing:

$$
\left(\mathcal{E}_{X} \cup\left\{y_{i}=\overline{1}\right\} \cup\left\{y_{j}=\overline{0} \mid y_{j} \in Y \backslash\left\{y_{i}\right\}\right\}\right) \text { stmt }\left\{y=a_{i}\right\} .
$$

3.2.4 Semirings with Multiplicative Inverse. A semiring may have multiplicative inverses: for each $\overline{0} \neq s \in S^{4}$ there exists $s^{-1} \in S$ such that $s \otimes s^{-1}=s^{-1} \otimes s=\overline{1}$. Examples of such semirings include $(\max ,+),(\min ,+),(\max , \times)$, and $(\min , \times)$.

The existence of multiplicative inverses is, however, insufficient for determining $a_{i}$. We therefore also assume the existence of a special value $S \ni z \neq \overline{0}$ that is similar to $\overline{0}$, namely, $z \oplus s=s$ for sufficiently many $s \in S$. For example, a very small value in semiring $(\max ,+)$ is a candidate for $z$.

${ }^{4}$ Unless $\overline{0}=\overline{1}$, we cannot define the inverse of $\overline{0}$ because no $\overline{0}^{-1} \in S$ can satisfy both $\overline{0}^{-1} \otimes \overline{0}=\overline{0}$ and $\overline{0}^{-1} \otimes \overline{0}=\overline{1}$. 
The existence of the special value $z$ enables us to infer $a_{i}=w \otimes z$, where $w$ is obtained by executing:

$$
\left(\mathcal{E}_{X} \cup\left\{y_{i}=z^{-1}\right\} \cup\left\{y_{j}=\overline{0} \mid y_{j} \in Y \backslash\left\{y_{i}\right\}\right\}\right) \text { stmt }\{y=w\} .
$$

This inference is at least reasonable, as the following reasoning indicates.

$$
\begin{aligned}
& \left(a_{0} \oplus\left(a_{1} \otimes \overline{0}\right) \oplus \cdots \oplus\left(a_{i} \otimes z^{-1}\right) \oplus \cdots \oplus\left(a_{k} \otimes \overline{0}\right)\right) \otimes z \\
= & \left(a_{0} \otimes z\right) \oplus a_{i} \\
= & \left(a_{0} \otimes z\right) \oplus\left(a_{0} \otimes a_{0}^{-1} \otimes a_{i}\right) \\
= & a_{0} \otimes\left(z \oplus\left(a_{0}^{-1} \otimes a_{i}\right)\right) \\
\approx & a_{0} \otimes\left(a_{0}^{-1} \otimes a_{i}\right) \\
= & a_{i}
\end{aligned}
$$

This somewhat doubtful inference is sufficient for our purpose. Our approach based on reverse engineering is conceptually unsound; hence, the inference of the coefficient is not necessarily sound.

3.2.5 Reduction without Multiplications. A reduction loop often consists of one kind of reduction operator. In this case, $a_{i}$ is $\overline{0}$ if reduction variable $y$ is independent of $y_{i}$ and $\overline{1}$ otherwise. Therefore, a standard value dependence analysis enables coefficients to be determined.

A value dependence analysis based on reverse engineering is presented in Section 4.1 .

3.2.6 Limitation. The approach we developed for inferring coefficients applies to most practically important semirings; however, a few semirings are excluded. An example is a (non-commutative) semiring over languages, $\left(2^{\Sigma^{*}}, \cup, \bullet, \emptyset,\{\varepsilon\}\right)$, where $\Sigma^{*}$ is the set of all finite strings over alphabet $\Sigma, X \bullet Y=\{v w \mid v \in X, w \in Y\}$, and $\varepsilon$ is the empty string. It is not a distributive lattice and has neither additive inverses nor multiplicative inverses.

\subsection{Computational Complexity}

The computational cost of the proposed approach depends on the number of variables, the number of candidate semirings, and the number of tests. Let $n$ be the number of tests, and suppose that the cost of evaluating stmt is $\mathrm{O}(|X|)$. The inference of a coefficient and a linear polynomial respectively takes time $\mathrm{O}(|X|)$ and $\mathrm{O}(|X||Y|)$. Therefore, the total cost of the parallelization process is $\mathrm{O}\left(n|X||Y|^{2}\left|\mathcal{S}_{\mathcal{R}}\right|\right)$. This cost should be reasonable unless the loop contains a huge number of variables.

Note that the number of tests actually run is much less than $n$ on average because a semiring inappropriate for the loop is rejected after only a few tests. Hence, complex loops for which most semirings are rejected tend to take less time. This is an interesting characteristic of the proposed approach.

\subsection{Code Generation}

A parallel program can be generated if an appropriate semiring is found. The code generation process is based on the

$$
\begin{array}{ll} 
& y_{1}=\overline{0} \\
y_{1}=\overline{0} & \vdots \\
\vdots & y_{i}=\overline{1} \\
y_{k}=\overline{0} & \vdots \\
\text { stmt } & y_{k}=\overline{0} \\
a_{0}=y & \text { stmt } \\
& a_{i}=a_{0}^{-1} \oplus y
\end{array}
$$

Figure 4. Code templates for calculating coefficients for the linear polynomial for reduction variable $y$.

existing semiring-based methods [25, 31, 38] except for the calculation of the coefficients.

The code for calculating the coefficients follows the approach described in Section 3.2 and uses variables instead of specific values. Figure 4 shows two examples. The code on the left is for calculating the constant coefficient, $a_{0}$. That on the right is for calculating $a_{i}$ when the semiring has additive inverses. The generated code contains many copies of stmt. We expect standard optimizations will remove most of the redundancy.

\section{Auxiliary Methods}

The approach we have developed is made applicable to more loops by the use of auxiliary methods.

\subsection{Loop Decomposition}

A loop may contain many operators that do not fit in a semiring. As an example, consider the following program, which checks whether a given string consists of well-structured parentheses [7, 18].

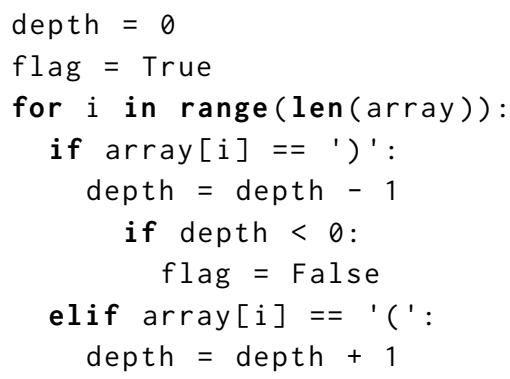

This loop contains two reduction variables, depth and flag. The former counts the nested parentheses, while the latter remembers whether there is an unmatched closing parenthesis. The program does not correspond to a semiring because the reduction variables are of different types.

Loop decomposition makes reduction parallelization easier $[10,27,28,31]$. For instance, the program above can be decomposed as follows. 


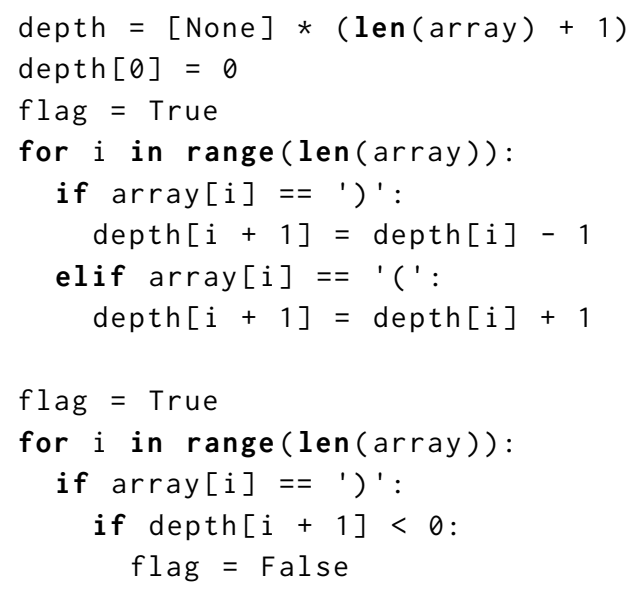

The first loop calculates the value of depth for every loop iteration and stores it in an array, and then the second one uses it to calculate flag. Each loop consists of one kind of calculation and hence can be expressed as a linear polynomial over a semiring. Although the first loop is somewhat different from typical reduction loops, it can be parallelized using the parallel scan algorithm (also known as the parallel prefix sum algorithm), whose details are described elsewhere [2].

This loop can be decomposed because depth does not depend on flag. A symbolic approach to analyzing such value dependences is common. Here we present an alternative reverse-engineering approach to value dependence analysis.

From the perspective of reduction parallelization, this kind of loop decompositions is necessary only when a loop contains more than one reduction variable which does not share a semiring in common. Nevertheless, in the rest of this subsection, we try to decompose a loop as much as possible. We will discuss recomposition of unnecessary decompositions in Section 4.2.

As did in Section 3, we consider a loop for which the body is stmt. Let $X$ be the set of variables. Their dependences are analyzed using the following algorithm.

1. Let $D=\emptyset$.

2. Do the following for each $x_{i}, x_{j} \in X$ :

a. Repeat the following test for a sufficient number of times. If it fails, add $x_{i} \rightarrow x_{j}$ to $D$ and go to Step 2 .

i. Let $\mathcal{E}=\left\{x_{k}=v_{k} \mid x_{k} \in X\right\}$, where each $v_{k}$ is a randomly generated value, and find $w$ such that $\mathcal{E}$ stmt $\left\{x_{j}=w\right\}$ by executing stmt.

ii. Let $\mathcal{E}^{\prime}=\mathcal{E} \backslash\left\{x_{i}=v_{i}\right\} \cup\left\{x_{i}=v_{i}^{\prime}\right\}$, where $v_{i}^{\prime} \neq v_{i}$ is another randomly generated value, and find $w^{\prime}$ such that $\mathcal{E}^{\prime}$ stmt $\left\{x_{j}=w^{\prime}\right\}$ by executing stmt.

iii. Check $w=w^{\prime}$.

3. Return the transitive closure of $D$.

This algorithm checks, for each pair of variables $x_{i}$ and $x_{j}$, whether a modification on $x_{i}$ affects the value of $x_{j}$. If so, it concludes that $x_{j}$ depends on $x_{i}$. The final step, i.e., taking the transitive closure, is necessary for correctly dealing with the following situation.

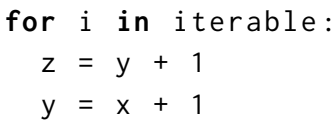

While the algorithm calculates $D=\{\mathrm{x} \rightarrow \mathrm{y}, \mathrm{y} \rightarrow \mathrm{z}\}$ for this loop, $\mathrm{z}$ depends on $\mathrm{x}$ via the loop-carried dependence of $\mathrm{y}$.

In addition to loop decomposition, the dependence analysis facilitates reduction parallelization in two ways. First, it detects reduction variables, which are self-dependent variables. Second, it enables the detection of loops that violate our prerequisites for parallelization, e.g., those that modify i terable inside the loop body.

\subsection{Loop Recomposition}

While loop decomposition improves parallelizability, it degenerates the performance of the parallel code because the parallel scan algorithm is much more complicated. Therefore, loop decomposition should be applied only when necessary.

Our semiring-based approach provides a natural guideline. Two consecutive loops can be composed without loss of parallelizability if they can be expressed by the same semiring. Therefore, by using the following algorithm, we can introduce the minimum number of loop decompositions.

1. Decompose the loop as many times as possible in accordance with the value dependence.

2. For each loop $l_{i}$, enumerate all the semirings that can parallelize $l_{i}$.

3. Recompose loops so that all the loops composed into one share a semiring in common and the number of resulting loops is minimized.

Considering all the appropriate semirings is important for loop recomposition. Consider the following example.

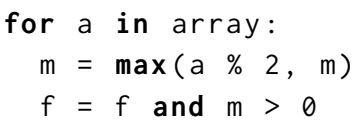

The reductions for $m$ and $f$ can be apparently parallelized by using max and $\wedge$, respectively. However, to avoid loop decomposition, we should be aware that the computation for $m$ can be expressed by $\vee$ as well.

\subsection{Parallelizing Nested Loops}

Since our approach regards the loop body as a black box, it can be applied to any complex loop. Nevertheless, in practice, it is desirable to use a specialized method to deal with nested loops. First, identifying the input-output behaviors for nested loops is time-consuming. Second, it is important to carefully determine which loop to parallelize. Parallelizing the outermost one may be impossible or result in slower code than parallelizing an inner one. A desirable strategy is to analyze each loop independently and derive a guideline for parallelization. Our approach leads to such a modular parallelization of nested loops. 
For explanation, we consider the following program. Note that our approach can deal with any type of nested loop.

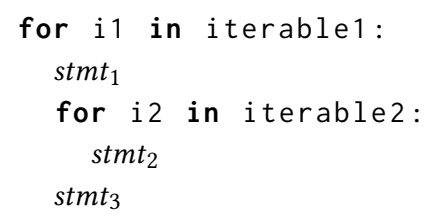

4.3.1 Parallelizability Detection and Code Generation. We apply the proposed approach (Section 3) to each of $s t m t_{1}, s t m t_{2}$, and stmt $t_{3}$ and then decide the code generation strategy based on the analysis results. Parallelizing the outer loop, i.e., evaluating each iteration for the i terable1 element in parallel, is possible if stmt ${ }_{1}, s_{t m} t_{2}$, and stmt $t_{3}$ can be expressed by the same semiring because their summaries (i.e., linear polynomials) can be merged. This approach is useful, especially if i terable1 is sufficiently long. Another possibility is to parallelize the inner loop. This approach requires stmt $t_{2}$ to correspond to linear polynomials (regardless of stmt $_{1}$ or stmt $_{3}$ ).

Consider the following specific example of a loop, which calculates the maximum sum of consecutive rows that contain the last row [8].

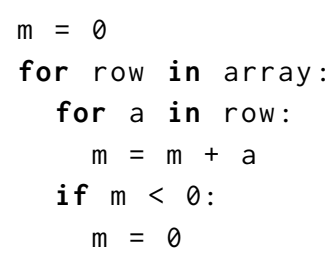

The body of the inner loop in this program uses + , whereas the conditional statement of the outer one corresponds to max. Because $(\max ,+)$ forms a semiring, both can be parallelized in our approach.

4.3.2 Value Dependence Analysis. The value dependence analysis described in Section 4.1 can be extended to nested loops in a modular manner. The algorithm is simple: analyze dependences for each of stmt $t_{1}, s t m t_{2}$, and stmt $t_{3}$ and return (the transitive closure of) their union. That is, two variables are dependent if they are dependent in $s t m t_{1}, s t m t_{2}$, or $\mathrm{stmt}_{3}$.

This algorithm is conservative. For the following program, it concludes that $s$ is self-dependent and thus a reduction variable even for the outer loop.

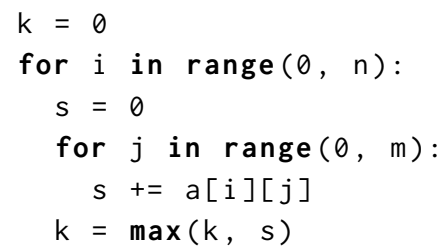

Nevertheless, we expect that this false detection is less serious compared with the advantages of its modularity.

\subsection{Array Access Index Inference}

Some reduction loops use arrays for storing calculated values. For example, array $r$ is updated in the following program, which is the inner loop for the longest common subsequence problem [8, 31],

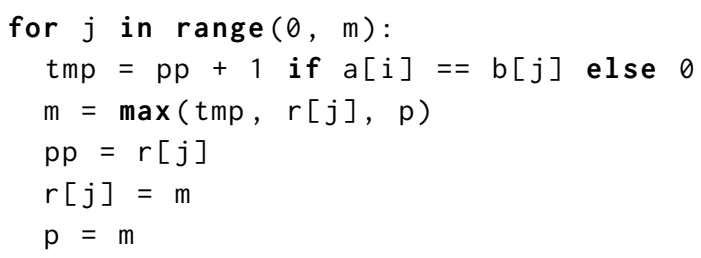

Our approach can be applied to this loop by regarding $r[j]$ as a "reduction variable", by virtue of the parallel scan algorithm, if the array is written in order. To achieve this, we must know how the array is accessed and which locations (i.e., array indices) should be regarded as reduction variables. Such information is typically gathered using symbolic analysis. Once again, reverse engineering can be used to infer this information.

We assume that each accessed location can be represented by a linear polynomial over $(+, \times)$. For each array $x$, we apply the following analysis. For simplicity, we assume that each execution of the loop body reads and writes $x$ at most once. Let $X$ be the set of variables that may affect the accessed locations.

1. Infer the coefficients of the linear polynomials for index-calculation expression by using the method described in Section 3.2. The set of indeterminates is $X$. Accessed locations for each execution are specified from the input-output behaviors as follows.

- $x[i]$ is written if the execution of the loop body modifies the value of $x[i]$.

- $x[i]$ is read if a modification on $x[i]$ affects the output of the loop body.

2. By random testing, verify that the inferred linear polynomials are correct. If not, the analysis fails.

The algorithm guesses the index-calculation expression by using the coefficient inference method we developed. The key is the method for inferring accessed locations, which is, in essence, similar to the value dependence analysis described in Section 4.1.

Recall the loop for the longest common subsequence problem. Observations of the accessed locations when $j=0$ and $j=1$ results in the suggestion of $0+1 \times j$ as a candidate. Since this passes the tests, $r[j]$ is regarded as a reduction variable.

\section{Use Case Scenarios}

The proposed reverse-engineering approach is inherently unsound. It may judge non-parallelizable loops as parallelizable. It thus appears to be unacceptable as a compiler optimization. 
We here demonstrate its applicability through potential use case scenarios.

\subsection{Use in Combination with Other Approaches}

A natural possibility is to use the proposed approach as a subprocedure for parallelization. That is, it is used to obtain a candidate of parallel implementation, and another method is then used to verify its correctness. This combination approach enables program synthesis to be reduced to program verification, which is generally easier. Moreover, the intensive use of random testing leads to fewer invocations of the verification method. This structure is similar to oracleguided program synthesis [17], which combines a candidate synthesizer and a verifier. The bottleneck of oracle-guided program synthesis is commonly the verifier, and thus, the synthesizer carefully generates candidates to minimize the invocation of the verifier.

In general, the standard forward-engineering approach, which uses program texts, and one based on reverse engineering are complementary. The former is commonly useful for syntactically simple programs, whereas the latter is useful for programs whose behaviors are simple. For example, the former may wrongly detect value dependence from $x$ to $\mathrm{y}$ in the following program.

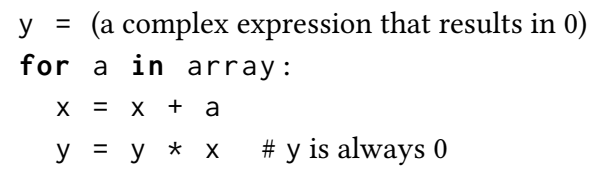

On the other hand, the latter may not detect value dependence from $x$ to $y$ in the following program.

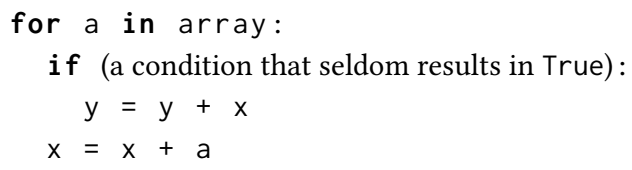

Combining the two approaches should thus be beneficial.

\subsection{Parallelization without Correctness Guarantee}

The soundness is sometimes overlooked for advanced optimizations. Some change numeric errors (e.g., optimizing $x=a * a * a * a$ to $y=a * a ; x=y * y)$. For the user-defined reduction operators in MPI ${ }^{5}$, programmers are responsible for verifying reduction operator associativity. Generally, it is relatively reasonable to require the programmer, as a part of "programming", to check the prerequisite of an optimization invoked in accordance with programmer directives or nonstandard compiler options. A similar situation arises in approximate computing [24], a branch of computing techniques to trade off accuracy for efficiency. Profile-guided inexact approaches to automatic loop parallelization can be found in QuickStep [23] and HELIX-UP [4].

\footnotetext{
${ }^{5}$ https: //www.mpi-forum.org/docs/
}

The reverse-engineering approach is (probabilistically) correct if the program behaves uniformly for any input. From the perspective mentioned above, it may be reasonable to require programmers to verify this uniformness. For example, our approach is applicable when the programmer knows that the reduction loop can be parallelized but does not want to actually write the parallel code.

\subsection{Speculative Parallelization}

Another approach that tolerates unsoundness is speculation. A speculative optimization is performed by assuming its correctness, and a fallback procedure is used when the result is found to be incorrect.

Our approach can be used for speculative parallelization. A thread (say, the main thread) sequentially evaluates the reduction loop as usual. Idle threads attempt parallelization in accordance with the input-output behaviors, which may be obtained from the main thread, and if likely possible, perform parallel reduction and then proceed to the next computation. The main thread continues its sequential evaluation and finally checks whether its result is the same as the one obtained by the parallel reduction. If the results differ, it stops the speculative computations of the other threads and restarts the computation from the current position using its result. This approach cannot achieve parallel speedup if the reduction is the only computation. However, a parallel reduction is commonly a part of the overall computation. A typical pattern is to gather global information necessary for the subsequent local tasks. For such patterns, the speculative approach would be beneficial.

The speculative approach is fully dynamic and hence suitable for JIT compilation, for which neither powerful constraint solvers nor program verifiers can be used. Moreover, it may be able to parallelize more loops than typical static approaches. For instance, consider the following loop.

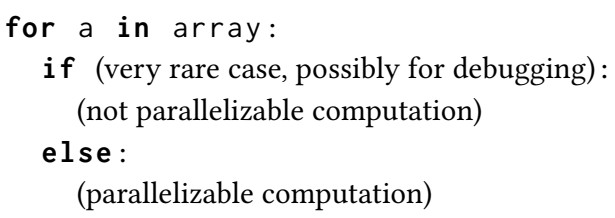

Static approaches commonly fail to parallelize this loop because they cannot exclude the possibility of nonparallelizable computation. However, the speculative approach ignores rare cases because random testing will probably not encounter them. This parallelization is beneficial unless the computation unfortunately contains a rare case.

\section{Experimental Evaluation}

We demonstrate the effectiveness of the proposed approach experimentally with our proof-of-concept implementation. 
Table 1. Results of detecting parallelizability of flat loops

\begin{tabular}{|c|c|c|c|}
\hline Benchmark program & Decomposition & Operator & Elapsed time (s) \\
\hline summation $[7,9,10,28,36]$ & & + & 0.02 \\
\hline summation of even elements [9] & & + & 0.02 \\
\hline summation of positives [9] & & + & 0.02 \\
\hline average $[7,9]$ & $\checkmark$ &,++ & 0.05 \\
\hline count positives [9] & & + & 0.02 \\
\hline count 1s [9] & & + & 0.03 \\
\hline count gaps [18] & $\checkmark$ & + & 0.04 \\
\hline maximum $[7,9,10,36]$ & & $\max$ & 0.03 \\
\hline second maximum [9] & $\checkmark$ & $\max , \max$ & 0.15 \\
\hline absolute maximum [9] & & $\max$ & 0.04 \\
\hline minimum $[7,9]$ & & $\min$ & 0.02 \\
\hline second minimum $[7,18]$ & $\checkmark$ & $\min$ & 0.05 \\
\hline maximum-minimum difference [9] & $\checkmark$ & $\max , \min$ & 0.08 \\
\hline count maximum elements [9] & $\checkmark$ & $\max ,+$ & 0.07 \\
\hline count minimum elements [9] & $\checkmark$ & $\min ,+$ & 0.05 \\
\hline dot product [36] & & + & 0.03 \\
\hline Hamming distance[7] & & + & 0.02 \\
\hline polynomial $[7,18,31]$ & $\checkmark$ & $(+, \times),+$ & 0.04 \\
\hline complex product $[36]$ & & $(+, \times)$ & 0.04 \\
\hline double exponential smoothing [18] & & $(+, \times)$ & 0.04 \\
\hline tridiagonal LU decomposition [31] & $\checkmark$ & $(+, \times)$ & $0.04^{*}$ \\
\hline finite difference method [31] & & $(+, \times)$ & 0.07 \\
\hline maximum length of continuous 1s [7] & $\checkmark$ &,$+ \max$ & 0.08 \\
\hline maximum gap between 1 s $[9,18]$ & &,$+ \max$ & 0.07 \\
\hline maximum sum between 0s [9] & &,$+ \max$ & 0.08 \\
\hline maximum prefix sum $[7,18,28]$ & $\checkmark$ &,$+ \max$ & 0.09 \\
\hline maximum suffix sum $[18,31]$ & $\checkmark$ & $(\max ,+)$ & 0.05 \\
\hline maximum segment sum $[7,9,10,18,28,31]$ & $\checkmark$ & $(\max ,+), \max$ & 0.29 \\
\hline maximum segment product [18] & $\checkmark$ & $(\max , \times), \max$ & 0.15 \\
\hline all same[9] & $\checkmark$ & $\wedge$ & 0.03 \\
\hline same numbers of $0 \mathrm{~s}$ and $1 \mathrm{~s}$ [9] & & + & 0.03 \\
\hline bracket matching $[7,18]$ & $\checkmark$ &,$+ \wedge$ & 0.04 \\
\hline visibility check [28] & $\checkmark$ & $\max$ & 0.08 \\
\hline dropwhile negative [7] & $\checkmark$ & $\vee$ & 0.04 \\
\hline find $1[9]$ & $\checkmark$ & $\vee$ & 0.03 \\
\hline sorted $[7,9]$ & $\checkmark$ & $\wedge$ & 0.03 \\
\hline $0 * 1^{*}[7]$ & $\checkmark$ & $\wedge$ & 0.03 \\
\hline$(01)^{*}[9]$ & $\checkmark$ & $\wedge, \wedge$ & 0.03 \\
\hline no 0 except the head [9] & & $\wedge$ & 0.02 \\
\hline no 0 except after $1[7]$ & $\checkmark$ & $\wedge, \wedge$ & 0.03 \\
\hline count matches of $1^{*}[9]$ & $\checkmark$ &,++ & 0.07 \\
\hline count matches of $1^{*} 2[9]$ & $\checkmark$ &,++ & 0.06 \\
\hline count matches of $10^{*} 2[9]$ & $\checkmark$ &,,+++ & 0.12 \\
\hline count matches of $1^{*} 2^{*} 3[9]$ & $\checkmark$ &,,+++ & 0.14 \\
\hline count matches of $10^{*} 20^{*} 3[9]$ & $\checkmark$ &,,+++ & 0.27 \\
\hline
\end{tabular}

\subsection{Implementation}

The input program consists of Python functions corresponding to the loop bodies and the types of their arguments and results. The types are numbers, Boolean values, and lists of numbers. To avoid the difficulty of equality checking caused by numeric errors, we used integers and floating-point numbers that do not introduce numeric errors (e.g., 0.5, 0.25, and 0.75 ). To express constraints over the inputs to the loop 
Table 2. Results of detecting parallelizability of nested loops

\begin{tabular}{|c|c|c|c|}
\hline Benchmark program & Decomposition & Operator & Elapsed time (s) \\
\hline 2D summation [8] & & + & 0.03 \\
\hline $2 \mathrm{D}$ sorted $[8]$ & $\checkmark$ & $\wedge$ & 0.03 \\
\hline 4D maximum-element index [36] & $\checkmark$ & $\max$ & 0.24 \\
\hline vertical sorted $[8]$ & & $\wedge$ & 0.01 \\
\hline diagonal sorted [8] & & $\wedge$ & 0.02 \\
\hline vertical increasing range [8] & $\checkmark$ & $\max , \wedge$ & 0.11 \\
\hline vertical overlapping range [8] & $\checkmark$ & $\max , \min , \wedge$ & 0.24 \\
\hline vertical decreasing range [8] & $\checkmark$ & $\max , \min , \wedge$ & 0.24 \\
\hline intersection of row ranges [8] & & $\wedge$ & 0.01 \\
\hline maximum of row minimums [8] & $\checkmark$ & $\min , \max$ & 0.07 \\
\hline maximum of column minimums [8] & $\checkmark$ & $\min , \max$ & 0.11 \\
\hline saddle point $[8]$ & $\checkmark$ & $\min , \max , \min , \max$ & 0.29 \\
\hline $2 \mathrm{D}$ maximum prefix sum [8] & $\checkmark$ &,$+ \max$ & 0.06 \\
\hline 2D maximum suffix sum [8] & & $(\max ,+)$ & 0.02 \\
\hline 2D maximum segment sum [8] & $\checkmark$ & $(\max ,+), \max$ & 0.05 \\
\hline maximum left-upper segment sum [8] & $\checkmark$ &,,$++ \max$ & 0.12 \\
\hline maximum right-lower segment sum [8] & $\checkmark$ & $+,(\max ,+), \max$ & 0.24 \\
\hline maximum right-upper segment sum [8] & $\checkmark$ & $+,(\max ,+), \max$ & 0.12 \\
\hline 3D maximum prefix sum $[8]$ & $\checkmark$ &,$+ \max$ & 0.06 \\
\hline 3D maximum suffix sum [8] & & $(\max ,+)$ & 0.01 \\
\hline 3D maximum segment sum [8] & $\checkmark$ & $(\max ,+), \max$ & 0.07 \\
\hline 3D maximum left-prefix sum [8] & $\checkmark$ &,,,$+++ \max$ & 0.19 \\
\hline count bracket-matching rows [8] & $\checkmark$ & $+, \wedge,+$ & 0.09 \\
\hline mode $[8]$ & $\checkmark$ &,$+ \max$ & 0.15 \\
\hline maximum difference of two arrays [8] & $\checkmark$ & $\max$ & 0.05 \\
\hline farthest matching of brackets [8] & $\checkmark$ & $+, \wedge, \max$ & 0.36 \\
\hline longest common subsequence $[8,31]$ & & $(\max ,+)$ & 0.08 \\
\hline independent elements [9] & & & $\mathrm{N} / \mathrm{A}$ \\
\hline 2D histogram [36] & & & $\mathrm{N} / \mathrm{A}$ \\
\hline
\end{tabular}

body, we used assert statements. If an AssertionError is caught during random testing, different inputs are generated; however, if the error occurs during the coefficient inference process, the semiring is rejected.

Our implementation consists of about 350 lines of Python code. It executes the input program by the exec function. We implemented the parallelizability detection (Section 3), the loop decomposition (Section 4.1), and the nested-loop parallelization (Section 4.3), which tries to parallelize the outermost loop. We incorporated neither the loop recomposition (Section 4.2), array access index inference (Section 4.4), nor any other analysis. We prepared the following semirings: $(+, \times),(\max ,+),(\max , \min ),(\min , \max ),(\wedge, \vee),(\vee, \wedge)$, and $(\max , \times)$.

We implemented two optimizations that improved the performance by a constant factor. Note that they did not enable otherwise impossible parallelization.

First, the system detects reduction variables that deliver values without any modification. Recall that the cost of our approach depends on the number of semirings that can parallelize the computation because random testing quickly rejects unsuccessful ones. The value-delivery variables can match any semiring and thus become a source of inefficiency. The system detects them by counting the numbers of $\overline{1}$ and $\overline{0}$ in the coefficients. If exactly one coefficient is $\overline{1}$ and the others are $\overline{0}$, it concludes without further analysis that all semirings can parallelize the computation.

Second, for programs that consist of multiple loops, every loop is tested in turn. This optimization enables quick rejection of semirings that are not suitable for one of the loops. The same approach is taken for detecting value dependences.

\subsection{Experimental Setting}

The experimental environment was a laptop PC equipped with an Intel Core i7-10510U CPU (1.80 GHz) and 8-GB memory running Ubuntu 20.04 OS on Windows Subsystem Linux (Windows 10) and CPython 3.8.3 was used to run our system. 
The elapsed time of running our system depends on the number of trials of random testing. We tested 1,000 inputs for each semiring and each reduction variable.

We exhaustively collected all the benchmark programs used in the previous studies on automatic parallelization of complex reduction loops $[7-10,18,28,31,36]^{6}$. If there was a diversity of programs, we adopted the most complex one. For example, the "maximum segment sum" program used here calculates not only the maximum sum but also the start and end indices of the maximum-sum segment.

Except for the "tridiagonal LU decomposition" program discussed later, we wrote the benchmark programs without considering parallelization. We did not intentionally use semiring operators. In particular, we often used conditionals instead of max and min operators.

\subsection{Experimental Results}

Tables 1 and 2 summarize the results. The "decomposition" column shows whether the loop was decomposable. The "operator" column shows the inferred semirings. If the loop contained only a single type of operator, the tables show the operator instead of a semiring. Semirings for value-delivery variables are omitted. If the loop was decomposable, the tables list the semirings for each loop; for example, the "average" program was decomposed into two loops, each of which used the + operator. The "elapsed time" column shows the time spent by the system.

When the system detected more than one semiring, Tables 1 and 2 show only the most intuitive one. The determination of reduction operators from multiple candidates may be practically important. Since our approach is unsound, the results of the system is sometimes wrong. We manually checked all the results and found no errors.

For all the benchmarks, the elapsed times were less than a second. As the system runs on the CPython interpreter, the performance seems acceptable.

The system could not parallelize two benchmarks, "independent elements" and "2D histogram". They require the $(\cup, \cap)$ semiring over sets and the addition operator over bit vectors, respectively. They should be parallelized once these operators are implemented, because the semirings either form a distributive lattice (Section 3.2.3) or have the additive inverses (Section 3.2.2).

The "tridiagonal LU decomposition" program was not immediately parallelized because it contains divisions in addition to additions and multiplications. We manually applied the transformation of Sato and Iwasaki [31], which facilitates the parallelization of loops containing divisions by introducing additional reduction variables. Moreover, in the process of coefficient detection, supplying 0 to a reduction

\footnotetext{
${ }^{6}$ We fixed a bug in the "maximum of row minimums" program [8], which introduced an additional conditional branch and thereby made parallelization more difficult.
}

variable caused a zero-division error, which was independent of the computation of the current reduction variables. We eliminated the error by manual loop decomposition.

In summary, the proposed approach could potentially parallelize all the benchmarks. This result demonstrates the usefulness of linear polynomials over semirings. Moreover, the manual program modifications necessary for parallelization were modest. In short, the use of reverse engineering makes parallelization efficient and robust.

\subsection{Unsuccessful Cases}

All the benchmark programs from the literature have fallen in the (potentially) successful cases of the proposed approach. To evaluate the unsuccessful cases of our approach, we develop the following two categories of negative examples supposed to fail.

One is the case where loop bodies cannot be expressed by any semiring detectable with our method (Section 3.2). For example, aggregation by using logarithm (Figure 5), summation with rounding (Figure 6), and summation with an absolute-value operator (Figure 7) fall in this category. These computations do not satisfy associativity.

The other is the case where syntactic structures hinder parallelization. For our approach, this situation is only due to reduction variables because they are the only syntactic information used in our parallelization. We have already mentioned to the case of the "tridiagonal LU decomposition" program, for which naive program is not successfully parallelized. Another example is the "maximum segment product" program [18]. To parallelize it successfully, its reduction variable should store not the minimum negative product but its absolute value because the $(\max , \times)$ semiring is defined over non-negative numbers.

Note that even these negative examples can be parallelized if certain invariants on their inputs hold. For instance, rounding is not problematic if it does not change the value. Similarly, the summation with absolute value operator and the maximum segment product should be parallelizable if all the array elements (and thereby the values of the reduction variables) are non-negative.

Table 3 shows the experimental results on the negative examples mentioned above. The "(w/ assertion)" in the program name means that we manually added an assertion of the invariant enabling parallelization. The $\emptyset$ symbol denotes that no semiring was detected.

The system successfully rejected all the negative examples. Manual assertions enabled the parallelization of more programs. However, the "rounding" program was unsuccessful even with the assertion because the coefficient inference algorithm assigned 1 of the additive identity to the reduction variable, contradicting the desired invariant of $(x+a[i])$ $\% 4==0$. 
Table 3. Results of detecting parallelizability of negative examples

\begin{tabular}{lccc}
\hline Benchmark program & Decomposition & Operator & Elapsed time (s) \\
\hline logarithm & & $\emptyset$ & 0.00 \\
rounding & & $\emptyset$ & 0.00 \\
rounding (w/ assertion) & & $\emptyset$ & 0.00 \\
summation with abs & & $\emptyset$ & 0.00 \\
summation with abs (w/ assertion) & & + & 0.03 \\
naive tridiagonal LU decomposition & $\checkmark$ & $\emptyset$ & 0.04 \\
maximum segment product with negative minimum & $\checkmark$ & $(\max , \times), \emptyset$ & 0.09 \\
maximum segment product with negative minimum (w/ assertion) & $\checkmark$ & $(\max , \times), \max$ & 0.67 \\
\hline
\end{tabular}

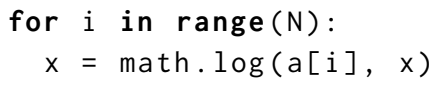

Figure 5. Reduction by logarithm.

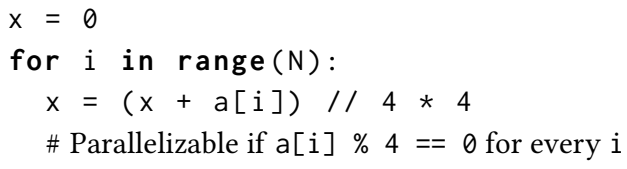

Figure 6. Summation with rounding.

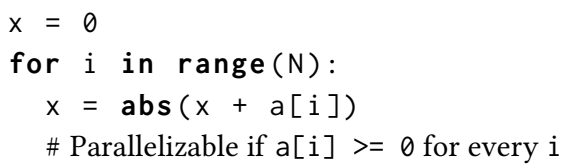

Figure 7. Summation with absolute-value operator.

\section{Related Work}

\subsection{Parallelization of Complex Reductions}

Numerous methods have been proposed [7-11, 15, 18, 22, 27, 28, 30, 31, 36, 38, 39] for the automatic parallelization of complex reductions. Our reverse-engineering approach is distinctive. We here compare our approach with closely related ones.

The existing methods that use linear polynomials over a semiring $[15,22,25,31,38]$ build on symbolic execution or abstract interpretation for analyzing computational structures and thus tend to fail to parallelize programs that do not explicitly use semiring operators. In particular, they have difficulty dealing with maximum (and minimum) computations expressed using conditionals. To reduce the difficulty, Sato and Iwasaki [31] proposed a method for extracting maximum operators from conditionals. It estimates candidates of maximum expressions and then verifies them by using an SMT solver. Unfortunately, such verification is generally time-consuming. Moreover, it assumes the normalization of programs for detecting maximum-like conditionals and thus is sensitive to details of conditional structures.
Jiang et al. [18] presented a method for the parallel evaluation of the sequential loops of conditional linear recurrences. It constructs linear polynomials at runtime from input-output samples obtained by executing a given sequential loop. This is similar to our coefficient inference in the use of input-output behaviors. However, their method is inherently speculative. If samples are insufficient for constructing linear polynomials, costly rollbacks are needed at runtime. Moreover, to guarantee the correctness of parallelization, their method requires the target loop bodies to pass a symbolic analysis, which restricts the usage of operators and conditionals.

Fisher and Ghuloum [10] pioneered in using program synthesis for the parallelization of complex reductions. Their method generates a template, which is a program with indeterminates, and checks, by heuristic rewriting, whether a composition of two templates can be simplified to one. Morihata and Matsuzaki [27] formulated a similar process using quantifier elimination. Farzan and Nicolet [7] used a syntax-guided program synthesis technique instead of heuristic rewriting.

Methods based on program synthesis techniques commonly suffer less from code variation and build upon more general formalizations, which can potentially subsume more instances. However, they are rather complicated and costly. Enumerative search depends heavily on heuristics to find desirable programs. Constraint solving techniques require leveraging dedicated solvers. Even with state-of-the-art heuristics and solvers, they are still questionable in terms of scalability against the complexity of input programs. Moreover, they commonly contain fragility against language features: heuristics often depend on syntax or idioms; constraint solving requires lifting programs completely to constraint formulae tractable for the underlying solvers.

Fedyukovich et al. [9] proposed a reduction parallelization method based on counter-example-guided inductive synthesis (CEGIS) [34] with multiple templates of parallel code. Their method is relatively robust against syntactic variations of input programs because it uses their specifications in the form of constraint formulae. Their approach is white-box in the sense that the constraint formulae capture the whole 
implementation of a given program. It does not focus on input-output behaviors for deliberate examples, in contrast to our reverse-engineering approach.

Farzan and Nicolet [8] proposed an automatic parallelization method for nested reduction loops. It reduces the parallelization of the outer loop to the possibility of summarizing the computations of the inner ones without knowing the values of the reduction variables. It is somewhat similar to our method, although restricted to the top-down analysis. In our proposed approach, every loop is independently analyzed, which enables more flexible code generation by virtue of the focus on linear polynomials over a semiring.

\subsection{Reverse Engineering in Program Synthesis}

The proposed approach is closely related to the programmingby-example paradigm [14], which synthesizes a program specified by a set of input-output examples. However, to the best of the authors' knowledge, the usefulness of reverse engineering in the context of program synthesis has not been explicitly remarked.

As discussed in Section 5.1, our approach can be regarded as a variant of oracle-guided program synthesis (OGS) [17]. OGS employs the I/O oracle, which generates input-output examples, and the verification oracle, which checks whether a synthesized candidate is a solution. In most previous studies, the I/O oracle was simply a set of input-output examples (or a programmer providing examples), and the verification oracle was an off-the-shelf constraint solver. Our approach combines reverse engineering with OGS. The given sequential program is adopted for the I/O oracle, candidate programs are constructed from carefully sampled input-output behaviors, and random testing is used as a verification oracle.

Ideas related to reverse engineering, particularly sampling of input-output behaviors, have been discussed in the literature on program synthesis and related topics. Podgurski and Pierce [29] proposed behavior sampling, which compares the input-output behaviors of software components to detect the reusability. Gulwani et al. [13] proposed a method for automatically solving ruler/compass-based geometry construction problems, where problem solving is reduced to random testing. Godefroid and Taly [12] automatically generated symbolic executions of processor instructions by observing their behaviors on a set of inputs. Biondi et al. [1] analyzed the input-output behaviors of an obfuscated program and used the programming-by-example paradigm to its deobfuscation. Le et al. [19] presented a method for automatic bug fixing, in which candidate repairs are generated from error-free input-output behaviors.

More recently, Collie et al. [6] developed a method for synthesizing a program from a black-box oracle, i.e., an executable software component whose internal structure is unknown. From the randomly-sampled input-output behaviors and type signature, their method infers probabilistic distribution of candidate templates in accordance with models learned from prior successful synthesis. Then, it synthesizes a program by instantiating the candidate templates using CEGIS. Our approach shares with theirs the idea of treating a given program as a black-box. A distinctive feature of our reverse-engineering approach is the use of deliberate sampling dedicated to the objective.

As seen from these previous studies, a reverse-engineering approach is useful for various applications. The current study adds a nontrivial application.

\subsection{Dynamic Approaches to Parallelization}

The reverse-engineering approach has an aspect of dynamic approaches, which intensively use runtime information. There have been several studies on dynamic approaches to reduction parallelization for overcoming unpredictables such as indirect memory accesses.

Rauchwerger and Padua [30] proposed a method for speculatively parallelizing reduction loops. It validates the correctness of parallel reduction by checking whether the reduction variable is updated by only a specified reduction operator. This correctness criterion is relaxed by Han et al. [16]. Wang et al. [37] similarly enhanced static reduction detection by using profile information.

Yu and Rauchwerger [39] proposed a method for selecting appropriate algorithms for parallel reductions dynamically and adaptively. It is independent of discovering parallelizable reductions. Our approach can use a similar method for improving the scheduling of parallelized reduction loops, especially loop nests.

Surendran et al. [35] proposed a method for synthesizing conditionally parallel function calls, which create parallel tasks only when the expected performance benefit exceed the overhead at runtime. The benefit and overhead are estimated by a profile-based analysis.

Our approach of inferring array access indices is related to dynamic polyhedral analysis $[3,32,33]$, in which the objective is to infer array access patterns. The polyhedral analysis enables different loop transformations for improving locality and parallelism. Chowdhury et al. [5] used a similar analysis for generating a cache-efficient parallel implementation of dynamic programming. Parallelization with these methods is based on data access independence and cannot resolve loop-carried value dependences in complex reduction loops.

\section{Concluding Remarks}

We have developed an approach to automatic reduction parallelization. It intensively uses reverse engineering and detects whether a given loop body corresponds to linear polynomials over a semiring. It enables nested loops to be parallelized in a modular manner. We have experimentally demonstrated the powerfulness and efficiency of our method.

Our investigation has demonstrated a promise of the reverse engineering approach for detecting the parallelizability 
of various reduction loops. Nevertheless, there remains a considerable gap between the current study and pragmatic auto-parallelization. We recognize at least two major issues to bridge the gap.

First, it is necessary to make further consideration of usecase scenarios (Section 5) because a target scenario would dominantly affect technical requirements and design issues. For example, if we make combined use of the approach and off-the-shelf verifiers, generated code should be amenable for them. If we leave the correctness guarantee to the programmer, well-designed programming interfaces should be a key to programing support.

Second, it is crucial to combine our approach with compiler technology. Naive use of our approach alone may result in inefficient parallel code. The final code quality is generally decided by a combined effect of various compiler optimizations, such as emerging semiring-based optimizations [20]. When handling loops inhabiting existing programs, we may need additional techniques, such as privatization, for making parallelized loops consistent with their contexts. Lack of analysis may also degrade the capability of detecting parallelizability (Section 6.4). Note that this study has dared to apply our approach alone without using other techniques to clarify the powerfulness and versatility of our approach.

Throughout this study, we have got the impression that the concept of reverse engineering could be useful for other tasks of program optimization and program synthesis. We consider loop incrementalization [21] and algorithmic improvement on graph-processing parallel programs [26] to be potential applications.

\section{Acknowledgments}

The authors are grateful to the anonymous reviewers and the "shepherd", Tomofumi Yuki, for their instructive comments to improve the presentation. The authors acknowledge the discussion in the meeting of the Cooperative Research Project of the Research Institute of Electrical Communication, Tohoku University. This work of the second author was partially supported by JSPS KAKENHI Grant Number 18K18032.

\section{References}

[1] Fabrizio Biondi, Sébastien Josse, Axel Legay, and Thomas Sirvent. 2017. Effectiveness of synthesis in concolic deobfuscation. Comput. Secur. 70 (2017), 500-515. https://doi.org/10.1016/j.cose.2017.07.006

[2] Guy E. Blelloch. 1993. Prefix Sums and Their Applications. In Synthesis of Parallel Algorithms, John H. Reif (Ed.). Morgan Kaufmann Publishers, Chapter 1. https://doi.org/10.1184/R1/6608579.v1

[3] Juan Manuel Martinez Caamaño, Manuel Selva, Philippe Clauss, Artyom Baloian, and Willy Wolff. 2017. Full runtime polyhedral optimizing loop transformations with the generation, instantiation, and scheduling of code-bones. Concurr. Comput. Pract. Exp. 29, 15 (2017). https://doi.org/10.1002/cpe.4192

[4] Simone Campanoni, Glenn Holloway, Gu-Yeon Wei, and David Brooks. 2015. HELIX-UP: Relaxing program semantics to unleash parallelization. In Proceedings of the 13th Annual IEEE/ACM International Symposium on Code Generation and Optimization (CGO '15). IEEE, 235-245.
https://doi.org/10.1109/CGO.2015.7054203

[5] Rezaul Chowdhury, Pramod Ganapathi, Stephen L. Tschudi, Jesmin Jahan Tithi, Charles Bachmeier, Charles E. Leiserson, Armando SolarLezama, Bradley C. Kuszmaul, and Yuan Tang. 2017. Autogen: Automatic Discovery of Efficient Recursive Divide-\&-Conquer Algorithms for Solving Dynamic Programming Problems. ACM Trans. Parallel Comput. 4, 1 (2017), 4:1-4:30. https://doi.org/10.1145/3125632

[6] Bruce Collie, Jackson Woodruff, and Michael F. P. O’Boyle. 2020. Modeling Black-Box Components with Probabilistic Synthesis. In Proceedings of the 19th ACM SIGPLAN International Conference on Generative Programming: Concepts and Experiences, GPCE 2020, Jeff Gray (Ed.). ACM. https://doi.org/10.1145/3425898.3426952

[7] Azadeh Farzan and Victor Nicolet. 2017. Synthesis of divide and conquer parallelism for loops. In Proceedings of the 38th ACM SIGPLAN Conference on Programming Language Design and Implementation, PLDI 2017, Barcelona, Spain, June 18-23, 2017, Albert Cohen and Martin T. Vechev (Eds.). ACM, 540-555. https://doi.org/10.1145/3062341.3062355

[8] Azadeh Farzan and Victor Nicolet. 2019. Modular divide-and-conquer parallelization of nested loops. In Proceedings of the 40th ACM SIGPLAN Conference on Programming Language Design and Implementation, PLDI 2019, Phoenix, AZ, USA, June 22-26, 2019, Kathryn S. McKinley and Kathleen Fisher (Eds.). ACM, 610-624. https://doi.org/10.1145/3314221. 3314612

[9] Grigory Fedyukovich, Maaz Bin Safeer Ahmad, and Rastislav Bodík. 2017. Gradual synthesis for static parallelization of single-pass arrayprocessing programs. In Proceedings of the 38th ACM SIGPLAN Conference on Programming Language Design and Implementation, PLDI 2017, Barcelona, Spain, fune 18-23, 2017, Albert Cohen and Martin T. Vechev (Eds.). ACM, 572-585. https://doi.org/10.1145/3062341.3062382

[10] Allan L. Fisher and Anwar M. Ghuloum. 1994. Parallelizing complex scans and reductions. In Proceedings of the ACM SIGPLAN'94 Conference on Programming Language Design and Implementation (PLDI), Orlando, Florida, June 20-24, 1994. 135-146. https://doi.org/10.1145/178243. 178255

[11] Alfons Geser and Sergei Gorlatch. 1999. Parallelizing functional programs by generalization. Journal of Functional Programming 9, 6 (1999), 649-673. https://doi.org/10.1017/S0956796899003536

[12] Patrice Godefroid and Ankur Taly. 2012. Automated synthesis of symbolic instruction encodings from I/O samples. In ACM SIGPLAN Conference on Programming Language Design and Implementation, PLDI '12, Beijing, China - June 11 - 16, 2012, Jan Vitek, Haibo Lin, and Frank Tip (Eds.). ACM, 441-452. https://doi.org/10.1145/2254064.2254116

[13] Sumit Gulwani, Vijay Anand Korthikanti, and Ashish Tiwari. 2011. Synthesizing geometry constructions. In Proceedings of the 32nd ACM SIGPLAN Conference on Programming Language Design and Implementation, PLDI 2011, San fose, CA, USA, June 4-8, 2011, Mary W. Hall and David A. Padua (Eds.). ACM, 50-61. https://doi.org/10.1145/1993498. 1993505

[14] Sumit Gulwani, Oleksandr Polozov, and Rishabh Singh. 2017. Program Synthesis. Found. Trends Program. Lang. 4, 1-2 (2017), 1-119. https: //doi.org/10.1561/2500000010

[15] Mohammad R. Haghighat and Constantine D. Polychronopoulos. 1996. Symbolic Analysis for Parallelizing Compilers. ACM Trans. Program. Lang. Syst. 18, 4 (1996), 477-518. https://doi.org/10.1145/233561.233568

[16] Liang Han, Wei Liu, and James Tuck. 2010. Speculative parallelization of partial reduction variables. In Proceedings of the CGO 2010, The 8th International Symposium on Code Generation and Optimization, Toronto, Ontario, Canada, April 24-28, 2010, Andreas Moshovos, J. Gregory Steffan, Kim M. Hazelwood, and David R. Kaeli (Eds.). ACM, 141-150. https://doi.org/10.1145/1772954.1772975

[17] Susmit Jha, Sumit Gulwani, Sanjit A. Seshia, and Ashish Tiwari. 2010. Oracle-guided component-based program synthesis. In Proceedings of the 32nd ACM/IEEE International Conference on Software Engineering - Volume 1, ICSE 2010, Cape Town, South Africa, 1-8 May 2010, Jeff 
Kramer, Judith Bishop, Premkumar T. Devanbu, and Sebastián Uchitel (Eds.). ACM, 215-224. https://doi.org/10.1145/1806799.1806833

[18] Peng Jiang, Linchuan Chen, and Gagan Agrawal. 2018. Revealing parallel scans and reductions in recurrences through function reconstruction. In Proceedings of the 27th International Conference on Parallel Architectures and Compilation Techniques, PACT 2018, Limassol, Cyprus, November 01-04, 2018, Skevos Evripidou, Per Stenström, and Michael F. P. O’Boyle (Eds.). ACM, 10:1-10:13. https: //doi.org/10.1145/3243176.3243204

[19] Xuan-Bach D. Le, Duc-Hiep Chu, David Lo, Claire Le Goues, and Willem Visser. 2017. S3: syntax- and semantic-guided repair synthesis via programming by examples. In Proceedings of the 2017 11th foint Meeting on Foundations of Software Engineering, ESEC/FSE 2017, Paderborn, Germany, September 4-8, 2017, Eric Bodden, Wilhelm Schäfer, Arie van Deursen, and Andrea Zisman (Eds.). ACM, 593-604. https://doi.org/10.1145/3106237.3106309

[20] Guilherme Vieira Leobas and Fernando Magno Quintão Pereira. 2020 Semiring Optimizations: Dynamic Elision of Expressions with Identity and Absorbing Elements. In Proceedings of the 2020 ACM SIGPLAN International Conference on Object-Oriented Programming, Systems, Languages, and Applications, OOPSLA 2020, part of SPLASH 2020, David Grove (Ed.). ACM. https://doi.org/10.1145/3428199

[21] Yanhong A. Liu, Scott D. Stoller, Ning Li, and Tom Rothamel. 2005. Optimizing aggregate array computations in loops. ACM Transactions on Programming Languages and Systems 27, 1 (2005), 91-125. https: //doi.org/10.1145/1053468.1053471

[22] Kiminori Matsuzaki, Zhenjiang Hu, and Masato Takeichi. 2006. Towards automatic parallelization of tree reductions in dynamic programming. In SPAA 2006: Proceedings of the 18th Annual ACM Symposium on Parallel Algorithms and Architectures, Cambridge, Massachusetts, USA, July 30 - August 2, 2006, Phillip B. Gibbons and Uzi Vishkin (Eds.). ACM, 39-48. https://doi.org/10.1145/1148109.1148116

[23] Sasa Misailovic, Deokhwan Kim, and Martin C. Rinard. 2013. Parallelizing Sequential Programs with Statistical Accuracy Tests. ACM Trans. Embed. Comput. Syst. 12, 2s (2013), 88:1-88:26. https://doi.org/ $10.1145 / 2465787.2465790$

[24] Sparsh Mittal. 2016. A Survey of Techniques for Approximate Computing. ACM Comput. Surv. 48, 4 (2016), 62:1-62:33. https://doi.org/ $10.1145 / 2893356$

[25] Akimasa Morihata. 2019. Lambda calculus with algebraic simplification for reduction parallelization by equational reasoning. Proc. ACM Program. Lang. 3, ICFP (2019), 80:1-80:25. https://doi.org/10.1145/ 3341644

[26] Akimasa Morihata, Kento Emoto, Kiminori Matsuzaki, Zhenjiang Hu, and Hideya Iwasaki. 2018. Optimizing Declarative Parallel Distributed Graph Processing by Using Constraint Solvers. In Functional and Logic Programming - 14th International Symposium, FLOPS 2018, Nagoya, Japan, May 9-11, 2018, Proceedings (Lecture Notes in Computer Science, Vol. 10818), John P. Gallagher and Martin Sulzmann (Eds.). Springer, 166-181. https://doi.org/10.1007/978-3-319-90686-7_11

[27] Akimasa Morihata and Kiminori Matsuzaki. 2010. Automatic Parallelization of Recursive Functions Using Quantifier Elimination. In Functional and Logic Programming, 10th International Symposium, FLOPS 2010, Sendai, Japan, April 19-21, 2010. Proceedings (Lecture Notes in Computer Science, Vol. 6009), Matthias Blume, Naoki Kobayashi, and Germán Vidal (Eds.). Springer, 321-336. https://doi.org/10.1007/9783-642-12251-4_23
[28] Kazutaka Morita, Akimasa Morihata, Kiminori Matsuzaki, Zhenjiang $\mathrm{Hu}$, and Masato Takeichi. 2007. Automatic inversion generates divideand-conquer parallel programs. In Proceedings of the ACM SIGPLAN 2007 Conference on Programming Language Design and Implementation, San Diego, California, USA, fune 10-13, 2007, Jeanne Ferrante and Kathryn S. McKinley (Eds.). ACM, 146-155. https://doi.org/10.1145/ 1250734.1250752

[29] Andy Podgurski and Lynn Pierce. 1993. Retrieving Reusable Software by Sampling Behaviour. ACM Trans. Softw. Eng. Methodol. 2, 3 (1993), 286-303. https://doi.org/10.1145/152388.152392

[30] Lawrence Rauchwerger and David A. Padua. 1999. The LRPD test: speculative run-time parallelization of loops with privatization and reduction parallelization. IEEE Trans. Parallel Distributed Syst. 10, 2 (1999), 160-180. https://doi.org/10.1109/71.752782

[31] Shigeyuki Sato and Hideya Iwasaki. 2011. Automatic parallelization via matrix multiplication. In Proceedings of the 32nd ACM SIGPLAN Conference on Programming Language Design and Implementation, PLDI 2011, San fose, CA, USA, June 4-8, 2011, Mary W. Hall and David A. Padua (Eds.). ACM, 470-479. https://doi.org/10.1145/1993498.1993554

[32] Manuel Selva, Fabian Gruber, Diogo Sampaio, Christophe Guillon, Louis-Noël Pouchet, and Fabrice Rastello. 2020. Building a Polyhedral Representation from an Instrumented Execution: Making Dynamic Analyses of Nonaffine Programs Scalable. ACM Trans. Archit. Code Optim. 16, 4 (2020), 45:1-45:26. https://doi.org/10.1145/3363785

[33] Andreas Simburger, Sven Apel, Armin Größlinger, and Christian Lengauer. 2019. PolyJIT: Polyhedral Optimization Just in Time. Int. 7. Parallel Program. 47, 5-6 (2019), 874-906. https://doi.org/10.1007/ s10766-018-0597-3

[34] Armando Solar-Lezama. 2013. Program sketching. Int. f. Softw. Tools Technol. Transf. 15, 5-6 (2013), 475-495. https://doi.org/10.1007/s10009012-0249-7

[35] Rishi Surendran and Vivek Sarkar. 2016. Automatic parallelization of pure method calls via conditional future synthesis. In Proceedings of the 2016 ACM SIGPLAN International Conference on Object-Oriented Programming, Systems, Languages, and Applications, OOPSLA 2016, part of SPLASH 2016, Amsterdam, The Netherlands, October 30 - November 4, 2016, Eelco Visser and Yannis Smaragdakis (Eds.). ACM, 20-38. https://doi.org/10.1145/2983990.2984035

[36] Patricia Suriana, Andrew Adams, and Shoaib Kamil. 2017. Parallel associative reductions in Halide. In Proceedings of the 2017 International Symposium on Code Generation and Optimization, CGO 2017, Austin, TX, USA, February 4-8, 2017, Vijay Janapa Reddi, Aaron Smith, and Lingjia Tang (Eds.). ACM, 281-291. https://doi.org/10.1109/CGO.2017.7863747

[37] Zheng Wang, Georgios Tournavitis, Björn Franke, and Michael F. P. O'Boyle. 2014. Integrating profile-driven parallelism detection and machine-learning-based mapping. ACM Trans. Archit. Code Optim. 11, 1 (2014), 2:1-2:26. https://doi.org/10.1145/2579561

[38] Dana N. Xu, Siau-Cheng Khoo, and Zhenjiang Hu. 2004. PType System: A Featherweight Parallelizability Detector. In Programming Languages and Systems: Second Asian Symposium, APLAS 2004, Taipei, Taiwan, November 4-6, 2004. Proceedings (Lecture Notes in Computer Science, Vol. 3302), Wei-Ngan Chin (Ed.). Springer, 197-212. https://doi.org/10. 1007/978-3-540-30477-7_14

[39] Hao Yu and Lawrence Rauchwerger. 2006. An Adaptive Algorithm Selection Framework for Reduction Parallelization. IEEE Trans. Parallel Distributed Syst. 17, 10 (2006), 1084-1096. https://doi.org/10.1109/ TPDS.2006.131 